\title{
Pulmonary thromboendarterectomy for pulmonary hypertension linked to thalassemia
}

\author{
Mohammad Izzat ${ }^{1}$, hazem aljasem ${ }^{1}$, and Ahmad Izzat ${ }^{1}$ \\ ${ }^{1}$ Damascus University Faculty of Medicine
}

October 15, 2020

\begin{abstract}
Chronic pulmonary thromboembolism is one of the pathogenic mechanisms for the development of pulmonary arterial hypertension in thalassemic patients, but there are no current recommendations for the management of this complication. We report performing successful pulmonary thrombo-endarterectomy in a patient with chronic thromboembolic pulmonary hypertension linked to $\beta$-thalassemia, which was followed by substantial hemodynamic and functional improvements. This report supports the feasibility of pulmonary thromboendarterectomy in thalassemic patients, and highlights the need for a comprehensive evaluation for both the presence and cause of pulmonary arterial hypertension prior to determining the likelihood of surgical cure.
\end{abstract}

\section{Introduction}

Pulmonary arterial hypertension (PAH) is a possible complication and a significant source of morbidity and mortality in patients with thalassemia syndromes [1]. Pathogenic mechanisms of PAH in thalassemia are multifactorial. Chronic hemolysis, reduced nitric oxide bioavailability, chronic hypercoagulable states and various disease-directed therapies may play important roles in its development [2]. Even though chronic thromboembolic pulmonary hypertension $(\mathrm{CTEPH})$ is a well-recognized cause of PAH in patients with $\beta$ thalassemia, surgical management of CTEPH has only rarely been reported in such cases thus far $[3,4]$. We report here the case of a patient with $\beta$-thalassemia complicated with CTEPH who underwent successful surgical pulmonary thrombo-endarterectomy (PTE). Approval for writing this report was obtained from the local ethics committee of our institution, and obtaining individual consent was waived due to the retrospective nature of the study.

\section{Case Report}

This is the case of a 21 years-old male who was diagnosed with $\beta$-thalassemia major at the age of 8 months. He underwent splenectomy at the age of 10 years, and has been receiving monthly blood transfusions ever since. Three months prior to admission he presented to a peripheral emergency department with a sudden episode of dyspnea and central cyanosis, and was suspected to be infected with covid-19. Chest CT scan was made and ruled out any interstitial lung involvement, and revealed the presence of bilateral pulmonary emboli. Anticoagulation therapy with warfarin was started, but on follow-up the patient developed progressive exertional dyspnea, deteriorating to NYHA functional class III.

The patient was referred to our institution because of worsening symptoms. Physical examination showed clinical evidence of right heart failure and pulmonary hypertension, with a mild drop in arterial oxygen saturation (93\%). Echocardiography revealed a dilated right atrium (RA) which contained a large $47 \times 35 \times 16$ $\mathrm{mm}$ hyperechogenic mobile mass attached to the RA free wall near the orifice of the inferior vena cava. The right ventricle (RV) was severely dilated and dysfunctional, with tricuspid annular plane systolic excursion (TAPSE) of $13 \mathrm{~mm}$ and severe tricuspid regurgitation. Estimated systolic pulmonary arterial pressure (PAP) was $85 \mathrm{mmHg}$. Lung perfusion scintigraphy was not available at our center. Chest CT angiography showed 
bilateral defects in the pulmonary arteries consistent with bilateral chronic pulmonary thromboemboli (fig. $1)$.

On the basis of this evaluation, the diagnosis of CTEPH was established and PTE was performed, the technique of which was reported previously [5]. Total circulatory arrest time was 45 minutes and total cardiopulmonary bypass perfusion time was 94 minutes. A large organized clot was removed from the RA, in addition to removing the thickened intima and organized thrombi from lobar and segmental pulmonary arteries of the left middle and lower lobes, as well as from all major branches on the right.

Early recovery following surgery was uneventful, and the patient was discharged from ICU on the $4^{\text {th }}$ postoperative day. Pre-discharge echocardiogram confirmed satisfactory hemodynamic results, with an improved RV function and an estimated PAP of $45 \mathrm{mmHg}$. The patient was discharged home 19 days after surgery on warfarin but no requirement for supplemental oxygen. Three months after discharge, significant improvements in clinical status (NYHA class I) and oxygen saturation (99\%) were noted despite a degree of residual pulmonary hypertension (PAP of $42 \mathrm{mmHg}$ ).

\section{Comment}

Valuable advances have been made in understanding the risk factors and mechanisms responsible for the development of PAH in thalassemic patients. It is now known that chronic hemolysis with the resulting nitric oxide depletion can lead to endothelial dysfunction and vascular remodeling and, in due course, to PAH [1]. Additionally, vascular thromboembolic occlusions can also develop due to in situthrombosis as well as to pulmonary thromboembolism attributable to the well-recognized hypercoagulable state [2]. The relative contribution of hemolysis-related vasculopathy versus pulmonary thromboembolism in the development of $\mathrm{PAH}$ in a specific patient depends on the severity of the genetic defect and the provided treatment methods (such as transfusion rates, chelation therapy and splenectomy) [1]. This needs to be evaluated in every case individually employing a detailed and systematic analysis of lung perfusion [6].

Conventional management approaches to PAH in thalassemic patients include hemoglobinopathy-targeted therapy, PAH-specific medications as well as general supportive treatment. Existing evidence indicates that currently available drug therapies only temporarily and sporadically improve symptoms in CTEPH, and remain ineffective in ameliorating the mechanical obstructions of the pulmonary arteries or in improving patients' survival [7]. In this context, given that PTE has been established as the gold-standard therapy for CTEPH, it is conceivable that PTE may comprise the only potentially curative therapy for CTEPH in thalassemic patients.

Experience to date with PTE in thalassemic patients with CTEPH is limited to small series or isolated case reports, nevertheless these reports favor PTE as an effective treatment of surgically accessible CTEPH $[3,4]$. We reported here the successful performance of PTE surgery in a thalassemic patient with CTEPH irresponsive medical treatment. Pre-operative assessment indicated that this patient was an appropriate candidate for PTE despite a slightly increased potential surgical risk owing to the underlying thalassemia. The post-operative course was relatively uneventful, and was coupled with an early substantial improvement in hemodynamics and functional status.

It is notable that the post-operative reduction in PAP in our patient was less than that commonly expected following PTE [3] which is likely to be due to the multifactorial nature of PAH in thalassemia. In fact, concern remains that the chronic hemolysis-related vasculopathy in the distal vessels may place thalassemic patients at increased risk of residual or recurrent PAH after PTE [4]. Therefore, long-term results of PTE in thalassemic patients remains to be elucidated through long-term follow-up. It is noteworthy here that balloon pulmonary angioplasty has recently been proposed as a potential new therapeutic option for this process [8].

In conclusion, this case report supports the feasibility of PTE in thalassemic patients with surgically accessible CTEPH. Given the complexity of the pathophysiology of PAH in thalassemia, a comprehensive evaluation for both the presence and cause of PAH is imperative prior to determining the likelihood of surgical cure in 
each individual patient.

\section{Author contributions}

* Mohammad Bashar Izzat: Concept and study design, data interpretation, and writing the manuscript.

* Hazem Aljasem: Data interpretation and approval of manuscript.

* Ahmad Walid Izzat: Data interpretation and approval of manuscript.

\section{References}

1. Fraidenburg DR, Machado RF. Pulmonary hypertension associated with thalassemia syndromes. Ann NY Acad Sci 2016; 1368:127-139

2. Eldor A, Rachmilewitz EA. The hypercoagulable state in thalassemia. Blood 2002; 99:36-43

3. Mahesh B, Besser M, Ravaglioli A, et al . Pulmonary endarterectomy is effective and safe in patients with haemoglobinopathies and abnormal red blood cells: the Papworth experience. Eur J CardioThorac Surg 2016; 50:537-541

4. Roggero S, Vullo S, Volpe G, Piga A, Albera C. Management of pulmonary arterial hypertension associated to thalassemia: when pulmonary endarterectomy is the best therapeutical option? A case report. J Thromb Thrombolysis 2015; 39:139-43

5. Jamieson SW, Auger WR, Fedullo PF, et al . Experience and results with 150 pulmonary thromboendarterectomy operations over a 29-month period. J Thorac Cardiovasc Surg 1993; 106:116-127

6. Simonneau G, Robbins IM, Beghetti M, et al . Updated clinical classification of pulmonary hypertension. J Am Coll Cardiol 2009; 54(1 Suppl):S43-54

7. Scholzel BE, Post MC, Plokker HT, Snijder RJ. Clinical worsening during long-term follow-up in inoperable chronic thromboembolic pulmonary hypertension. Lung 2011; 190:161-167

8. Karyofyllis P, Tsiapras D, Papadopoulou V, et al . Balloon pulmonary angioplasty is a promising option in thalassemic patients with inoperable chronic thromboembolic pulmonary hypertension. J Thromb Thrombolysis 2018, 46:516-520

\section{Figure legends}

Fig. 1 - Chest CT angiography showing defects in the right (long arrows) and left (arrow heads) pulmonary arteries in keeping with bilateral chronic pulmonary thromboemboli. 


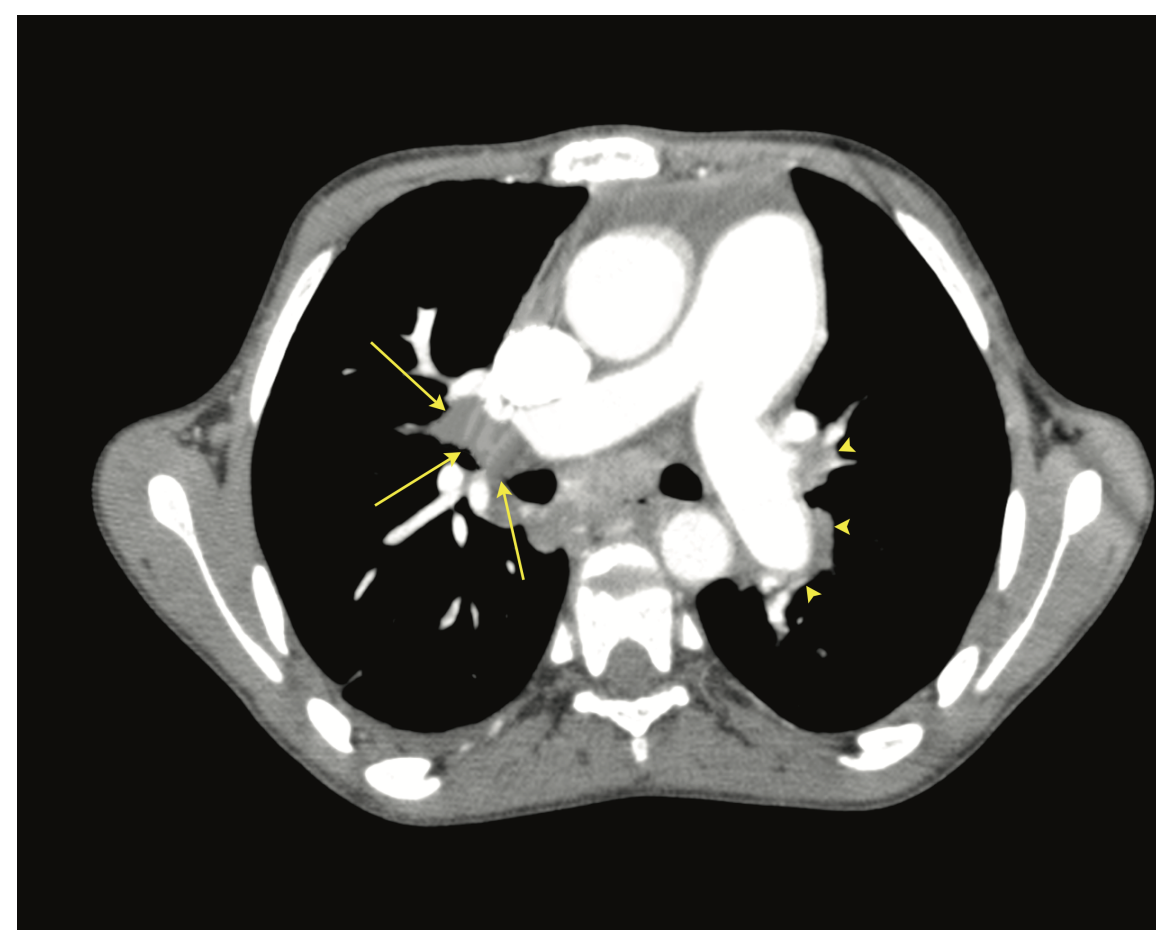

